\section{AROMATIC SUBSTITUTION}

\section{A different direction}

J.Am. Chem. Soc. 136, 4109-4112 (2014)

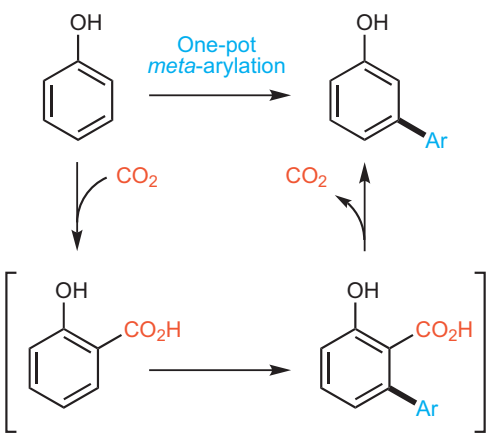

It is well understood that the outcome of substitution reactions on benzene rings depends on the substituents already present. In phenols, for example, additions to the ring system are directed to the ortho- and parapositions, relative to the hydroxyl group. This raises an interesting synthetic challenge: any phenol-derived synthetic target with a substituent in a meta- position must be made by a method that avoids functionalizing a phenol, often requiring lengthy synthetic sequences. Now, Igor Larrosa and co-workers from Queen Mary University of London have developed a one-pot sequence of reactions that allows arylation of unprotected phenols at the meta-position.

In a previous approach to this problem, a remote directing group that directs a palladium catalyst to the meta-position was installed on the phenol.This method, however, introduces additional synthetic steps in installing and subsequently removing the directing group. Larrosa and co-workers' method instead uses the known ortho/paradirecting effect of the phenol to install a transient carboxyl group onto the ring. This group subsequently directs a palladiumcatalysed cross-coupling reaction at the position that is ortho to the carboxyl group and thus meta to the hydroxyl substituent. Finally, decarboxylation of the arene affords selectively functionalized 1,3-substituted phenol derivatives. The process exhibits good tolerance to various substituents, including electron-donating and electron-withdrawing groups, and substitution patterns on either of the aryl coupling partners.

The elegance of this process comes at a price, however. High-pressure carbon dioxide is required to carry out the carboxylation step, and high temperatures are required throughout the sequence. But the economy of synthesis afforded by this process is shown by the synthesis of a $\gamma$-secretase inhibitor, a drug candidate for the treatment of Alzheimer's disease. The previous synthesis of this compound required eight synthetic steps and proceeded with an overall yield of $6 \%$. Starting instead with a meta-arylation of 5-bromophenol, the team prepared the target in just three steps with a yield of $41 \%$. $P M$

\section{DRUG DISCOVERY}

\section{Follow the pheromones' lead}

Angew. Chem. Int. Ed. http://doi.org/

f2qh85 (2014)

To find an exotic ingredient for a cooking project you could search in a huge supermarket, but a visit to a specialized food store might be quicker. Researchers are confronted with a similar problem when using combinatorial chemistry for drug discovery: a huge and diverse chemical library might harbour lead compounds for a specific target. But searching a small specialized library is more economical. Consequently, the question of how to design specialized chemical libraries is a matter of intense research. Now, Gisbert Schneider and co-workers from ETH Zürich have described a computational strategy for building such libraries.

The core of their method is an algorithm inspired by the way ants search for food: they fan out randomly but those that find food leave a pheromone trail. Other ants follow the trail and strengthen the connection when more food is found. But the pheromones evaporate if routes remain unused, which makes the algorithm adaptive. The algorithm works even if more than one optimization criterion has to be considered, a feature that Schneider and co-workers use to hunt a drug with activity against two targets involved in neuropsychiatric disorders - the sigma-1 and dopamine $\mathrm{D} 4$ receptors.

The starting point is an exhaustive list of amines and aldehydes/ketones. Randomly combining these in reductive amination yields 20 million possible products. But the ant algorithm allows them to home in on the useful ones. Equipped with bioactivities from a large database, the virtual ants assign scores to the product combinations they encounter. The scores reflect a compound's affinity and selectivity for the targets. At the end of the simulation, the final connection strength points the authors towards promising reactant combinations - islands of perfectly specialized chemical libraries in a sea of unusable structures. Analysis of these smaller libraries yields a few highly potent structures for which the bioactivity is experimentally verified. With an overall success rate of $90 \%$, the ant-inspired algorithm holds great promise for computational drug discovery.

$L M$

Written by Enda Bergin, Claire Hansell,

Paul MacLellan and Leonie Mueck.

\section{blogroll $^{\text {( }}$}

\section{Inviting ire and iron}

Flawed plastics testing and the chemistry of cranberries.

A toddler eyes the camera sternly while drinking from a sippy cup underneath the headline of a Mother Jones article that reads, 'The Scary New Evidence on BPA-free Plastics' (http://go.nature.com/ baAyvC). Sounds alarming. But it turns out that the research is actually three years old, which John Spevacek at It's the Rheo Thing points out, "hardly qualifies as new," and further, that "none of this evidence qualifies as evidence" (http://go.nature. com/O87J70).

The original research tested plastics for oestrogenic activity after subjecting them to unrealistically abusive conditions. This included exposure to UV light with unnaturally high energy and using an autoclave instead of a dishwasher. A test they had to run, suggests Spevacek wryly, "since dishwashers aren't available in the Austin, Texas area." Scare journalism based on misinformation is all too common, but those of us paying attention are grateful when someone with Spevacek's expertise takes the time to succinctly uncover faulty data.

Let's talk about a substance we don't have to be afraid of - cranberries. Highschool senior Meera Mody writes about the chemistry of this fruit (http://go.nature. $\mathrm{com} / \mathrm{btfLXa}$ ) on What's UR Rxn?, a group blog run by students at Detroit County Day School who make personal everyday connections to chemistry topics.

Mody notes that cranberries were used hundreds of years ago by Native Americans to treat infections, and modern studies have explained their antibacterial activity. Mody writes that "the phenolic ingredients in cranberries largely give them their healthy reputation" because polyphenols can bind to and remove excess iron, which reduces cellular oxidative stress. With a knack for relating chemistry in a clear and engaging way, these self-identified 'chemjournalists' are ones to watch.

Written by Tien Nguyen, who blogs at http://mustlovescience.com 